\title{
On a Generalization of Hilbert's Inequality and Its Applications
}

\author{
Baoju Sun (Corresponding author) \& Fuzhen Wu \\ Department of Mathematics, Zhejiang Water Conservancy \& Hydropower University \\ Hangzhou 310018, China \\ E-mail: sunbj@zjwchc.com
}

\begin{abstract}
In this paper, the author gives a new extension of Hilbert's inequality by introducing some parameters. Also its application has been taken into consideration.
\end{abstract}

Keywords: Hilbert's inequality, Hölder inequality, Homogeneous function

\section{Introduction}

If $a_{n} \geq 0, b_{n} \geq 0$, such that $0<\sum_{n=0}^{\infty} a_{n}^{2}<\infty, 0<\sum_{n=0}^{\infty} b_{n}^{2}<\infty$, then the famous Hilbert's inequality (Hardy, G. H.(1952)) is given by

$$
\sum_{m=0}^{\infty} \sum_{n=0}^{\infty} \frac{a_{m} b_{n}}{m+n+1}<\pi\left(\sum_{n=0}^{\infty} a_{n}^{2}\right)^{\frac{1}{2}}\left(\sum_{n=0}^{\infty} b_{n}^{2}\right)^{\frac{1}{2}}
$$

where the constant factor is the best possible.

Recently, some improvements and extensions of (1) are given in papers (Gao, M. Z. (1998), Kuang, J. C. (2000), Yang, B. C. (1999, 2002), Yang, B. C. and Debnath, L. $(1998,1999))$, We also have a classical extension of Hilbert's inequality with $(p, q)$-parameter form as follow:

If $p>1, \frac{1}{p}+\frac{1}{q}=1, a_{n} \geq 0, b_{n} \geq 0$, such that $0<\sum_{n=0}^{\infty} a_{n}^{p}<\infty, 0<\sum_{n=0}^{\infty} b_{n}^{q}<\infty$,then

$$
\sum_{m=0}^{\infty} \sum_{n=0}^{\infty} \frac{a_{m} b_{n}}{m+n+1}<\frac{\pi}{\sin (\pi / p)}\left(\sum_{n=0}^{\infty} a_{n}^{p}\right)^{\frac{1}{p}}\left(\sum_{n=0}^{\infty} b_{n}^{q}\right)^{\frac{1}{q}}
$$

where the constant factor $\pi / \sin (\pi / p)$ is the best possible ( Hardy, G. H. (1952)).

Ingham, A. E. (1936) has proved that if $a_{n} \geq 0,0<\sum_{n=0}^{\infty} a_{n}^{2}<\infty, \lambda>0$, then

$$
\sum_{m=0}^{\infty} \sum_{n=0}^{\infty} \frac{a_{m} a_{n}}{m+n+\lambda} \leq M(\lambda) \sum_{n=0}^{\infty} a_{n}^{2}
$$

where

$$
M(\lambda)=\left\{\begin{array}{cc}
\pi / \sin \lambda \pi, & 0<\lambda<1 / 2 \\
\pi, & \lambda \geq 1 / 2
\end{array}\right.
$$

Yang, B. C. (1999) proved that

$$
\sum_{m=0}^{\infty} \sum_{n=0}^{\infty} \frac{a_{m} b_{n}}{(m+n+1)^{t}}<B\left(\frac{p+t-2}{p}, \frac{q+t-2}{q}\right)\left\{\sum_{n=0}^{\infty}\left(n+\frac{1}{2}\right)^{1-t} a_{n}^{p}\right\}^{\frac{1}{p}}\left\{\sum_{n=0}^{\infty}\left(n+\frac{1}{2}\right)^{1-t} b_{n}^{q}\right\}^{\frac{1}{q}}
$$

where $B(u, v)$ is beta function, $p>1, \frac{1}{p}+\frac{1}{q}=1, a_{n} \geq 0, b_{n} \geq 0,2-\min \{p, q\}<t \leq 2$ and $0<\sum_{n=0}^{\infty}\left(n+\frac{1}{2}\right)^{1-t} a_{n}^{p}<\infty$, $0<\sum_{n=0}^{\infty}\left(n+\frac{1}{2}\right)^{1-t} b_{n}^{q}<\infty$

Yang, B. C. (2005) proved that 


$$
\sum_{m=0}^{\infty} \sum_{n=0}^{\infty} \frac{a_{m} b_{n}}{(m+n+1)^{t}}<B\left(\frac{p+t-2}{p}, \frac{q+t-2}{q}\right)\left\{\sum_{n=0}^{\infty}\left(n+\frac{1}{2}\right)^{(p-1)(2-t)-1} a_{n}^{p}\right\}^{\frac{1}{p}}\left\{\sum_{n=0}^{\infty}\left(n+\frac{1}{2}\right)^{(q-1)(2-t)-1} b_{n}^{q}\right\}^{\frac{1}{q}}
$$

Kuang, J. C.(2000) discussed the general series

$$
\sum_{m=0}^{\infty} \sum_{n=0}^{\infty} K(m+\lambda, n+\lambda) a_{m} b_{n}
$$

they gave the following theorem:

Theorem A Let $p>1, \frac{1}{p}+\frac{1}{q}=1, a_{n} \geq 0, b_{n} \geq 0$, and $0<\sum_{n=0}^{\infty}(n+\lambda)^{1-t} a_{n}^{p}<\infty, 0<\sum_{n=0}^{\infty}(n+\lambda)^{1-t} b_{n}^{q}<\infty$, $\frac{1}{2} \leq \lambda \leq \frac{1}{2} \min \{p, q\}$, and let $K(x, y)$ be nonnegative and homogeneous function of degree $-t(t>0)$. If $K(1, y)$ has first four derivatives continuous on $(0, \infty)$, and $(-1)^{n} K^{(n)}(1, y) \geq 0$, for $n=0,1,2,3,4, K^{(m)}(1, y) y^{-\frac{2 \lambda}{r}} \rightarrow 0, y \rightarrow \infty$, for $m=0,1, I(r, \lambda)=\int_{0}^{\infty} K(1, u) u^{-\frac{2 \lambda}{r}} d u<\infty, r=p, q$.then

$$
\sum_{m=0}^{\infty} \sum_{n=0}^{\infty} K(m+\lambda, n+\lambda) a_{m} b_{n}<\left\{\sum_{m=0}^{\infty}[I(q, \lambda)-\phi(q, m, t, \lambda)](m+\lambda)^{1-t} a_{m}^{p}\right\}^{\frac{1}{p}} \times\left\{\sum_{n=0}^{\infty}[I(p, \lambda)-\phi(p, n, t, \lambda)](n+\lambda)^{1-t} b_{n}^{q}\right\}^{\frac{1}{q}},
$$

where

$$
\phi(r, m, t, \lambda)=\left(\frac{\lambda}{\lambda+m}\right)^{1-\frac{2 \lambda}{r}}\left\{K\left(1, \frac{\lambda}{\lambda+m}\right)\left[\frac{1}{1-2 \lambda / r}-\frac{1}{2 \lambda}\left(1+\frac{1}{3 r}\right)\right]-\frac{1}{24 \lambda(\lambda+m)} K^{\prime}\left(1, \frac{\lambda}{\lambda+m}\right)\right\}>0
$$

and $r=p, q$.

The major objective of this paper is to formulate a new inequality related to the general series (6). which is an extension of (1), (5).

\section{Some lemmas}

Lemma 2.1 Let $f(x)$ have its first four derivatives continuous on $[0, \infty),(-1)^{n} f^{(n)}(x)>0 \quad(n=0,1,2,3,4), \int_{0}^{\infty} f(x) d x<$ $\infty, f(x), f^{\prime}(x) \rightarrow 0(x \rightarrow \infty)$, then

$$
\sum_{k=0}^{\infty} f(k)<\int_{0}^{\infty} f(x) d x+\frac{1}{2} f(0)-\frac{1}{12} f^{\prime}(0)
$$

The proof of Lemma 2.1 is given by Kuang, J. C.(2000).

Lemma 2.2 Let $p>1, \frac{1}{p}+\frac{1}{q}=1, \frac{1}{2} \leq \lambda \leq \frac{1}{2} \min \{p, q\}, 2-\frac{\min (p, q)}{2 \lambda}<t \leq 2, K(x, y)$ be nonnegative and homogeneous function of degree $-t$. If $K(1, y)$ has first four derivatives continuous on $(0, \infty)$, and , for $n=0,1,2,3,4, K^{(m)}(1, y) y^{-\frac{2 \lambda}{r}(2-t)} \rightarrow 0, y \rightarrow$ $\infty$, for $m=0,1 . I(r, \lambda)=\int_{0}^{\infty} K(1, u) u^{-\frac{2 \lambda}{r}(2-t)} d u<\infty, r=p, q$. Define the weight coefficient as:

$$
\omega(r, m, t, \lambda)=\sum_{n=0}^{\infty} K(m+\lambda, n+\lambda)\left(\frac{m+\lambda}{n+\lambda}\right)^{\frac{2 \lambda(2-t)}{r}}
$$

then

$$
\omega(r, m, t, \lambda)<(m+\lambda)^{1-t}[I(r, \lambda)-\phi(r, m, t, \lambda)] .
$$

Where

$$
\phi(r, m, t, \lambda)=\left(\frac{\lambda}{\lambda+m}\right)^{1-\frac{2 \lambda}{r}(2-t)}\left\{K\left(1, \frac{\lambda}{\lambda+m}\right)\left[\frac{1}{1-2 \lambda / r(2-t)}-\frac{1}{2 \lambda}\left(1+\frac{2-t}{3 r}\right)\right]-\frac{1}{24 \lambda(\lambda+m)} K^{\prime}\left(1, \frac{\lambda}{\lambda+m}\right)\right\}>0 .
$$

\section{Proof Let}

$$
f(x)=K(m+\lambda, x+\lambda)\left(\frac{x+\lambda}{m+\lambda}\right)^{-\frac{2 \lambda}{r}(2-t)}=(m+\lambda)^{-t} K\left(1, \frac{x+\lambda}{m+\lambda}\right)\left(\frac{x+\lambda}{m+\lambda}\right)^{-\frac{2 \lambda}{r}(2-t)},
$$


then

$$
\begin{aligned}
f^{\prime}(x)= & (m+\lambda)^{-t-1} K^{\prime}\left(1, \frac{x+\lambda}{m+\lambda}\right)\left(\frac{x+\lambda}{m+\lambda}\right)^{-\frac{2 \lambda}{r}(2-t)} \\
& -\frac{2 \lambda}{r}(2-t)(m+\lambda)^{-t-1} K\left(1, \frac{x+\lambda}{m+\lambda}\right)\left(\frac{x+\lambda}{m+\lambda}\right)^{-\frac{2 \lambda}{r}(2-t)-1} \\
f(0)= & (m+\lambda)^{-t} K\left(1, \frac{\lambda}{m+\lambda}\right)\left(\frac{\lambda}{m+\lambda}\right)^{-\frac{2 \lambda}{r}(2-t)}, \\
f^{\prime}(0)= & (m+\lambda)^{-t-1} K^{\prime}\left(1, \frac{\lambda}{m+\lambda}\right)\left(\frac{\lambda}{m+\lambda}\right)^{-\frac{2 \lambda}{r}(2-t)} \\
& -\frac{2 \lambda}{r}(2-t)(m+\lambda)^{-t-1} K\left(1, \frac{\lambda}{m+\lambda}\right)\left(\frac{\lambda}{m+\lambda}\right)^{-\frac{2 \lambda}{r}(2-t)-1} .
\end{aligned}
$$

By lemma 2.1 we have

$$
\begin{aligned}
\omega(r, m, t, \lambda)= & \sum_{k=0}^{\infty} f(k)<\int_{0}^{\infty} f(x) d x+\frac{1}{2} f(0)-\frac{1}{12} f^{\prime}(0) \\
= & \int_{0}^{\infty}(m+\lambda)^{-t} K\left(1, \frac{x+\lambda}{m+\lambda}\right)\left(\frac{x+\lambda}{m+\lambda}\right)^{-\frac{2 \lambda}{r}(2-t)} d x+\frac{1}{2}(m+\lambda)^{-t} K\left(1, \frac{\lambda}{m+\lambda}\right)\left(\frac{\lambda}{m+\lambda}\right)^{-\frac{2 \lambda}{r}(2-t)} \\
& -\frac{1}{12}(m+\lambda)^{-t-1} K^{\prime}\left(1, \frac{\lambda}{m+\lambda}\right)\left(\frac{\lambda}{m+\lambda}\right)^{-\frac{2 \lambda}{r}(2-t)}+\frac{\lambda}{6 r}(2-t)(m+\lambda)^{-t-1} K\left(1, \frac{\lambda}{m+\lambda}\right)\left(\frac{\lambda}{m+\lambda}\right)^{-\frac{2 \lambda}{r}(2-t)-1}, \\
\omega(r, m, t, \lambda)<(m+\lambda)^{1-t}\left[\int_{0}^{\infty} K(1, u) u^{-\frac{2 \lambda}{r}(2-t)} d u-\psi(r, m, t, \lambda)\right] & \\
& \psi(r, m, t, \lambda)=\int_{0}^{\frac{\lambda}{m+\lambda}} K(1, u) u^{-\frac{2 \lambda}{r}(2-t)} d u-\left(\frac{\lambda}{m+\lambda}\right)^{1-\frac{2 \lambda}{r}(2-t)}\left(\frac{1}{2 \lambda}+\frac{2-t}{6 r \lambda}\right) K\left(1, \frac{\lambda}{m+\lambda}\right) \\
& +\frac{1}{12 \lambda^{2}}\left(\frac{\lambda}{m+\lambda}\right)^{2-\frac{2 \lambda}{r}(2-t)} K^{\prime}\left(1, \frac{\lambda}{m+\lambda}\right) .
\end{aligned}
$$

Integrating by parts twice gives

$$
\begin{aligned}
& \int_{0}^{\frac{\lambda}{m+\lambda}} K(1, u) u^{-\frac{2 \lambda}{r}(2-t)} d u \\
& =\frac{1}{1-2 \lambda / r(2-t)} \int_{0}^{\frac{\lambda}{m+\lambda}} K(1, u) d u^{1-\frac{2 \lambda}{r}(2-t)} \\
& =\frac{1}{1-2 \lambda / r(2-t)}\left[K\left(1, \frac{\lambda}{m+\lambda}\right)\left(\frac{\lambda}{m+\lambda}\right)^{1-\frac{2 \lambda}{r}(2-t)}-\int_{0}^{\frac{\lambda}{m+\lambda}} K^{\prime}(1, u) u^{1-\frac{2 \lambda}{r}(2-t)} d u\right] \\
& =\frac{1}{1-2 \lambda / r(2-t)} K\left(1, \frac{\lambda}{m+\lambda}\right)\left(\frac{\lambda}{m+\lambda}\right)^{1-\frac{2 \lambda}{r}(2-t)} \\
& -\frac{1}{1-2 \lambda / r(2-t)} \cdot \frac{1}{2-2 \lambda / r(2-t)} \int_{0}^{\frac{\lambda}{m+\lambda}} K^{\prime}(1, u) d u^{2-\frac{2 \lambda}{r}(2-t)} \\
& =\frac{1}{1-2 \lambda / r(2-t)} K\left(1, \frac{\lambda}{m+\lambda}\right)\left(\frac{\lambda}{m+\lambda}\right)^{1-\frac{2 \lambda}{r}(2-t)} \\
& -\frac{1}{1-2 \lambda / r(2-t)} \cdot \frac{1}{2-2 \lambda / r(2-t)} K^{\prime}\left(1, \frac{\lambda}{m+\lambda}\right)\left(\frac{\lambda}{m+\lambda}\right)^{2-\frac{2 \lambda}{r}(2-t)} \\
& +\frac{1}{1-2 \lambda / r(2-t)} \cdot \frac{1}{2-2 \lambda / r(2-t)} \int_{0}^{\frac{\lambda}{m+\lambda}} K^{\prime \prime}(1, u) u^{2-\frac{2 \lambda}{r}(2-t)} d u \\
& >\frac{1}{1-2 \lambda / r(2-t)} K\left(1, \frac{\lambda}{m+\lambda}\right)\left(\frac{\lambda}{m+\lambda}\right)^{1-\frac{2 \lambda}{r}(2-t)} \\
& -\frac{1}{1-2 \lambda / r(2-t)} \cdot \frac{1}{2-2 \lambda / r(2-t)} K^{\prime}\left(1, \frac{\lambda}{m+\lambda}\right)\left(\frac{\lambda}{m+\lambda}\right)^{2-\frac{2 \lambda}{r}(2-t)} .
\end{aligned}
$$


From this and equality (11), we obtain

$$
\begin{aligned}
\psi(r, m, t, \lambda)> & {\left[\frac{1}{1-2 \lambda / r(2-t)}-\frac{1}{2 \lambda}\left(1+\frac{2-t}{3 r}\right)\right] K\left(1, \frac{\lambda}{m+\lambda}\right)\left(\frac{\lambda}{m+\lambda}\right)^{1-\frac{2 \lambda}{r}(2-t)} } \\
& -\left[\frac{1}{1-\frac{2 \lambda / r(2-t)}{2-2 \lambda / r(2-t)}} \cdot \frac{1}{12 \lambda^{2}}\right] K^{\prime}\left(1, \frac{\lambda}{m+\lambda}\right)\left(\frac{\lambda}{m+\lambda}\right)^{2-\frac{2 \lambda}{r}(2-t)} .
\end{aligned}
$$

Since $\frac{1}{2} \leq \lambda \leq \frac{1}{2} \min \{p, q\}$, we have

$$
2 \lambda \geq 1>\frac{3 r^{2}+r(2-t)}{3 r^{2}+3 r(2-t)+(2-t)^{2}}=\frac{r(3 r+2-t)}{3 r^{2}+3 r(2-t)+(2-t)^{2}},
$$

that is,

$$
\begin{gathered}
\frac{1}{2 \lambda}<\frac{3 r}{3 r+2-t}+\frac{2-t}{r}, \\
\frac{1}{2 \lambda}-\frac{2-t}{r}<\frac{3 r}{3 r+2-t}, \\
1-\frac{2 \lambda(2-t)}{r}<\frac{6 \lambda r}{3 r+2-t},
\end{gathered}
$$

So that

$$
\frac{1}{2 \lambda}\left(1+\frac{2-t}{3 r}\right)\left(1-\frac{2 \lambda}{r}(2-t)\right)<1
$$

This implies

$$
\frac{1}{1-2 \lambda / r(2-t)}-\frac{1}{2 \lambda}\left(1+\frac{2-t}{3 r}\right)>0
$$

Next note that $\frac{2}{r}(2-t)<\frac{1}{\lambda} \leq 2$, which implies that

$$
0<\left(\frac{1}{\lambda}-\frac{2(2-t)}{r}\right)\left(\frac{1}{\lambda}-\frac{2-t}{r}\right) \leq 4 .
$$

That is

$$
\begin{gathered}
\frac{1}{1-2 \lambda / r(2-t)} \cdot \frac{1}{2-2 \lambda / r(2-t)} \geq \frac{1}{8 \lambda^{2}} \\
\frac{1}{1-2 \lambda / r(2-t)} \cdot \frac{1}{2-2 \lambda / r(2-t)}-\frac{1}{12 \lambda^{2}} \geq \frac{1}{8 \lambda^{2}}-\frac{1}{12 \lambda^{2}}=\frac{1}{24 \lambda^{2}} .
\end{gathered}
$$

By hypotheses $K^{\prime}\left(1, \frac{\lambda}{m+\lambda}\right) \leq 0$, this and inequalities (12), (13), imply that

$$
\begin{aligned}
\psi(r, m, t, \lambda) & >\left(\frac{\lambda}{m+\lambda}\right)^{1-\frac{2 \lambda}{r}(2-t)}\left\{\left[\frac{1}{1-2 \lambda / r(2-t)}-\frac{1}{2 \lambda}\left(1+\frac{2-t}{3 r}\right)\right] K\left(1, \frac{\lambda}{m+\lambda}\right)-\frac{1}{24 \lambda} \cdot \frac{1}{m+\lambda} K^{\prime}\left(1, \frac{\lambda}{m+\lambda}\right)\right\} \\
& =\phi(r, m, t, \lambda)>0 .
\end{aligned}
$$

From (10), the lemma 2.2 is proved.

\section{Main results}

Theorem 3.1 Let $p>1, \frac{1}{p}+\frac{1}{q}=1, a_{n} \geq 0, b_{n} \geq 0, \frac{1}{2} \leq \lambda \leq \frac{1}{2} \min \{p, q\}, 2-\frac{\min (p, q)}{2 \lambda}<t \leq 2$, and $0<$ $\sum_{m=0}^{\infty}(m+\lambda)^{2 \lambda(p-2)(2-t)+1-t} a_{m}^{p}<\infty, 0<\sum_{n=0}^{\infty}(n+\lambda)^{2 \lambda(q-2)(2-t)+1-t} b_{n}^{q}<\infty, K(x, y)$ be nonnegative and homogeneous function of degree $-t$. If $K(1, y)$ has first four derivatives continuous on $(0, \infty)$, and $(-1)^{n} K^{(n)}(1, y) \geq 0$, for $n=0,1,2,3,4$; $K^{(m)}(1, y) y^{-\frac{2 \lambda}{r}(2-t)} \rightarrow 0, y \rightarrow \infty$,for $m=0,1$,

$$
I(r, \lambda)=\int_{0}^{\infty} K(1, u) u^{-\frac{2 \lambda}{r}(2-t)} d u<\infty, r=p, q .
$$


then

$$
\begin{aligned}
& \sum_{m=0}^{\infty} \sum_{n=0}^{\infty} K(m+\lambda, n+\lambda) a_{m} b_{n} \\
< & \left\{\sum_{m=0}^{\infty}[I(p, \lambda)-\phi(p, m, t, \lambda)](m+\lambda)^{2 \lambda(p-2)(2-t)+1-t} a_{m}^{p}\right\}^{\frac{1}{p}} \\
& \times\left\{\sum_{n=0}^{\infty}[I(q, \lambda)-\phi(q, n, t, \lambda)](n+\lambda)^{2 \lambda(q-2)(2-t)+1-t} b_{n}^{q}\right\}^{\frac{1}{q}},
\end{aligned}
$$

where

$\phi(r, m, t, \lambda)$

$$
=\left(\frac{\lambda}{\lambda+m}\right)^{1-\frac{2 \lambda}{r}(2-t)}\left\{K\left(1, \frac{\lambda}{\lambda+m}\right)\left[\frac{1}{1-2 \lambda / r(2-t)}-\frac{1}{2 \lambda}\left(1+\frac{2-t}{3 r}\right)\right]-\frac{1}{24 \lambda(\lambda+m)} K^{\prime}\left(1, \frac{\lambda}{\lambda+m}\right)\right\}>0
$$

and $r=p, q$.

Proof By Hölder inequality, we have

$$
\begin{aligned}
& \sum_{m=0}^{\infty} \sum_{n=0}^{\infty} K(m+\lambda, n+\lambda) a_{m} b_{n} \\
= & \sum_{m=0}^{\infty} \sum_{n=0}^{\infty}[K(m+\lambda, n+\lambda)]^{\frac{1}{p}}\left[\frac{(m+\lambda)^{\frac{1}{q^{2}}}}{(n+\lambda)^{\frac{1}{p^{2}}}}\right]^{2 \lambda(2-t)} a_{m} \cdot[K(m+\lambda, n+\lambda)]^{\frac{1}{q}}\left[\frac{(n+\lambda)^{\frac{1}{p^{2}}}}{(m+\lambda)^{\frac{1}{q^{2}}}}\right]^{2 \lambda(2-t)} b_{n} \\
\leq & \left\{\sum_{m=0}^{\infty} \sum_{n=0}^{\infty} K(m+\lambda, n+\lambda)\left[\frac{(m+\lambda)^{\frac{p}{q^{2}}}}{(n+\lambda)^{\frac{1}{p}}}\right]^{2 \lambda(2-t)} a_{m}^{p}\right\}^{\frac{1}{p}} \times\left\{\sum_{m=0}^{\infty} \sum_{n=0}^{\infty} K(m+\lambda, n+\lambda)\left[\frac{(n+\lambda)^{\frac{q}{p^{2}}}}{(m+\lambda)^{\frac{1}{q}}}\right]^{2 \lambda(2-t)} b_{n}^{q}\right\}^{\frac{1}{q}} \\
< & \left\{\sum_{m=0}^{\infty} \omega(p, m, t, \lambda)(m+\lambda)^{2 \lambda(p-2)(2-t)} a_{m}^{p}\right\}^{\frac{1}{p}} \times\left\{\sum_{n=0}^{\infty} \omega(q, n, t, \lambda)(n+\lambda)^{2 \lambda(q-2)(2-t)} b_{n}^{q}\right\}^{\frac{1}{q}},
\end{aligned}
$$

where

$$
\omega(r, m, t, \lambda)=\sum_{n=0}^{\infty} K(m+\lambda, n+\lambda)\left(\frac{m+\lambda}{n+\lambda}\right)^{\frac{2 \lambda(2-t)}{r}},
$$

thus, by (9), we have (16). Theorem 3.1 is proved.

Let $p>1, \frac{1}{p}+\frac{1}{q}=1, \frac{1}{2} \leq \lambda \leq \frac{1}{2} \min \{p, q\}, 2-\frac{\min (p, q)}{2 \lambda}<t \leq 2, t_{0}=2-\frac{\min (p, q)}{2 \lambda}$. Setting $g_{r}(t)=t+\frac{2 \lambda}{r}(2-t)-1, r=p, q$. then $g^{\prime}{ }_{r}(t)=1-\frac{2 \lambda}{r} \geq 0, g_{r}(t)$ is nondecreasing on $\left[t_{0}, 2\right], t_{0}<t \leq 2, g_{r}(t) \geq g_{r}\left(t_{0}\right)$. If $p \leq q, g_{p}\left(t_{0}\right)=2-\frac{p}{2 \lambda}+\frac{p}{p}-1 \geq 2-p \geq 0$; $g_{q}\left(t_{0}\right)=2-\frac{p}{2 \lambda}+\frac{p}{q}-1 \geq 2-p+p-2 \geq 0, g_{r}(t) \geq 0$. When $p>q, g_{r}(t) \geq 0$ is valid.

We take $K(x, y)$ as $K(x, y)=(x+y)^{-t}$, then

$$
I(r, \lambda)=\int_{0}^{\infty}(1+u)^{-t} u^{-\frac{2 \lambda}{r}(2-t)} d u=B\left(1-\frac{2 \lambda}{r}(2-t), t+\frac{2 \lambda}{r}(2-t)-1\right)
$$

$$
\begin{aligned}
\phi(r, m, t, \lambda) & =\left(\frac{\lambda}{\lambda+m}\right)^{1-\frac{2 \lambda}{r}(2-t)}\left\{\left(\frac{m+\lambda}{m+2 \lambda}\right)^{t}\left[\frac{1}{1-2 \lambda / r(2-t)}-\frac{1}{2 \lambda}\left(1+\frac{2-t}{3 r}\right)\right]+\frac{t(m+\lambda)^{t}}{24 \lambda(m+2 \lambda)^{t+1}}\right\} \\
& >\left(\frac{\lambda}{\lambda+m}\right)^{1-\frac{2 \lambda}{r}(2-t)} \frac{1}{2^{t}}\left[\frac{1}{1-2 \lambda / r(2-t)}-\frac{1}{2 \lambda}\left(1+\frac{2-t}{3 r}\right)\right] \\
& =h(r, m, t, \lambda) .
\end{aligned}
$$

Theorem 3.2 Let $p>1, \frac{1}{p}+\frac{1}{q}=1, a_{n} \geq 0, b_{n} \geq 0, \frac{1}{2} \leq \lambda \leq \frac{1}{2} \min \{p, q\}, 2-\frac{\min (p, q)}{2 \lambda}<t \leq 2$, and $0<$ 
$\sum_{m=0}^{\infty}(m+\lambda)^{2 \lambda(p-2)(2-t)+1-t} a_{m}^{p}<\infty, 0<\sum_{n=0}^{\infty}(n+\lambda)^{2 \lambda(q-2)(2-t)+1-t} b_{n}^{q}<\infty$, then

$$
\begin{aligned}
& \sum_{m=0}^{\infty} \sum_{n=0}^{\infty} \frac{a_{m} b_{n}}{(m+n+2 \lambda)^{t}} \\
< & \left\{\sum_{m=0}^{\infty}\left[B\left(1-\frac{2 \lambda}{p}(2-t), t+\frac{2 \lambda}{p}(2-t)-1\right)-h(p, m, t, \lambda)\right](m+\lambda)^{2 \lambda(p-2)(2-t)+1-t} a_{m}^{p}\right\}^{\frac{1}{p}} \\
\times & \left\{\sum_{n=0}^{\infty}\left[B\left(1-\frac{2 \lambda}{q}(2-t), t+\frac{2 \lambda}{q}(2-t)-1\right)-h(q, m, t, \lambda)\right](n+\lambda)^{2 \lambda(q-2)(2-t)+1-t} b_{n}^{q}\right\}^{\frac{1}{q}},
\end{aligned}
$$

where

$$
h(r, m, t, \lambda)=\left(\frac{\lambda}{\lambda+m}\right)^{1-\frac{2 \lambda}{r}(2-t)} \frac{1}{2^{t}}\left[\frac{1}{1-2 \lambda / r(2-t)}-\frac{1}{2 \lambda}\left(1+\frac{2-t}{3 r}\right)\right] .
$$

when $\lambda=1 / 2$, we have

Theorem 3.3 Let $p>1, \frac{1}{p}+\frac{1}{q}=1, a_{n} \geq 0, b_{n} \geq 0,2-\min (p, q)<t \leq 2$, and $0<\sum_{m=0}^{\infty}\left(m+\frac{1}{2}\right)^{(p-2)(2-t)+1-t} a_{m}^{p}<\infty, 0<$ $\sum_{n=0}^{\infty}\left(n+\frac{1}{2}\right)^{(q-2)(2-t)+1-t} b_{n}^{q}<\infty$, then

$$
\begin{aligned}
& \sum_{m=0}^{\infty} \sum_{n=0}^{\infty} \frac{a_{m} b_{n}}{(m+n+1)^{t}} \\
< & \left\{\sum_{m=0}^{\infty}\left[B\left(\frac{p+t-2}{p}, \frac{q+t-2}{q}\right)-\frac{1}{(2 m+1)^{\frac{p+t-2}{p}}} \frac{1}{2^{t}}\left(\frac{p}{p+t-2}-1-\frac{2-t}{3 p}\right)\right]\left(m+\frac{1}{2}\right)^{(p-2)(2-t)+1-t} a_{m}^{p}\right\}^{\frac{1}{p}} \\
& \times\left\{\sum_{n=0}^{\infty}\left[B\left(\frac{p+t-2}{p}, \frac{q+t-2}{q}\right)-\frac{1}{(2 n+1)^{\frac{q+t-2}{q}}} \frac{1}{2^{t}}\left(\frac{q}{q+t-2}-1-\frac{2-t}{3 q}\right)\right]\left(n+\frac{1}{2}\right)^{(q-2)(2-t)+1-t} b_{n}^{q}\right\}^{\frac{1}{q}} .
\end{aligned}
$$

Evidently, inequality (20) is an improvement of (5).

By taking $t=1$ in Theorem 3.3, we obtain

Theorem 3.4 Let $p>1, \frac{1}{p}+\frac{1}{q}=1, a_{n} \geq 0, b_{n} \geq 0$, and $0<\sum_{m=0}^{\infty}\left(m+\frac{1}{2}\right)^{p-2} a_{m}^{p}<\infty, 0<\sum_{n=0}^{\infty}\left(n+\frac{1}{2}\right)^{q-2} b_{n}^{q}<\infty$, then

$$
\begin{aligned}
& \sum_{m=0}^{\infty} \sum_{n=0}^{\infty} \frac{a_{m} b_{n}}{m+n+1} \\
< & \left\{\sum_{m=0}^{\infty}\left[\frac{\pi}{\sin (\pi / p)}-\frac{1}{2(2 m+1)^{\frac{1}{q}}}\left(q+\frac{1}{3 q}-\frac{4}{3}\right)\right]\left(m+\frac{1}{2}\right)^{p-2} a_{m}^{p}\right\}^{\frac{1}{p}} \\
& \times\left\{\sum_{n=0}^{\infty}\left[\frac{\pi}{\sin (\pi / p)}-\frac{1}{2(2 m+1)^{\frac{1}{p}}}\left(p+\frac{1}{3 p}-\frac{4}{3}\right)\right]\left(n+\frac{1}{2}\right)^{q-2} a_{n}^{q}\right\}^{\frac{1}{q}} .
\end{aligned}
$$

By taking $p=q=2$ in Theorem 3.4, we have

$$
\sum_{m=0}^{\infty} \sum_{n=0}^{\infty} \frac{a_{m} b_{n}}{m+n+1}<\left\{\sum_{m=0}^{\infty}\left[\pi-\frac{5}{12(2 m+1)^{\frac{1}{2}}}\right] a_{m}^{2}\right\}^{\frac{1}{2}}\left\{\sum_{n=0}^{\infty}\left[\pi-\frac{5}{12(2 n+1)^{\frac{1}{2}}}\right] a_{n}^{2}\right\}^{\frac{1}{2}} .
$$

\section{References}

Gao, M. Z. and Yang, B. C. (1998). On the extended Hilbert's inequality. Proceeding of the American Mathematical Society, 126, 751-759.

Hardy, G. H., Littlewood, J. E. and Pólya, G. (1952). Inequalities, Gambridge University Press, Gambridge. Ingham, A. E. (1936). A note on Hilbert's inequality. Journal of the London Mathematical Society, 11, $237-240$.

Kuang, J. C. and Debnath, L. (2000). On new generalizations of Hilbert's inequality and their applications. Journal of Mathematical Analysis and Applications, $245,248-265$. 
Yang, B. C. and Debnath, L. (1999). On a new generalization of Hardy Hilbert's inequality and its applications. Journal of Mathematical Analysis and Applications, 233, 484-497.

Yang, B. C. and Debnath, L. (1998). On a new strengthened Hardy-Hilbert's inequality. International Journal of Mathematics and Mathematical Sciences, 21:1, 403-408.

Yang, B. C. (1999). On a strengthened version of the more accurate Hardy-Hilbert's inequality. Acta Mathematica, Sinica (China), 42, 1103-1110.

Yang, B. C. (2002). On a generalization of Hardy-Hilbert's double series theorem. Mathematical Inequalities \& Applications, 5, 197-204.

Yang, B. C. (2005). On a strengthened Hardy-Hilbert's inequality and its generalization. Analysis Mathematica, 31, 151-161. 